\title{
Penile Malignant Peripheral Nerve Sheath Tumor
}

National Cancer Institute

\section{Source}

National Cancer Institute. Penile Malignant Peripheral Nerve Sheath Tumor. NCI

Thesaurus. Code C162584.

A rare malignant peripheral nerve sheath tumor that occurs in the penis. 